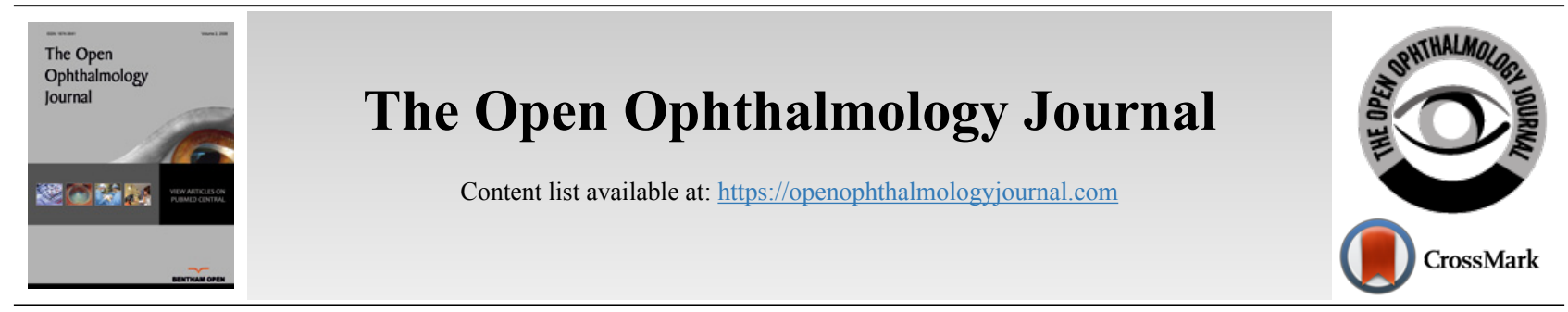

\title{
LETTER
}

\section{Inexpensive Binocular Indirect Ophthalmoscopy}

\author{
Aqeeda Singh ${ }^{1, *}$, Sheng C. Hong ${ }^{2}$ and Kelechi Ogbuehi ${ }^{2}$ \\ ${ }^{1}$ University of Otago Medical School, Dunedin, New Zealand \\ ${ }^{2}$ Dunedin Hospital, Southern District Health Board, Dunedin, New Zealand
}

\begin{abstract}
:
We aim to discuss an inexpensive method of conducting binocular indirect ophthalmoscopy without the binocular indirect ophthalmoscope headset. This technique only requires the examiner to hold a light source between their eyes and hold a condensing lens at an arm's length. This may be particularly valuable in resource-poor settings.
\end{abstract}

Keywords: Binocular indirect ophthalmoscopy, Inexpensive BIO, Ophthalmoscopy, Ophthalmic lenses, Retinal examination, Stereoscopic vision.

\begin{tabular}{|l|c|c|c|}
\hline Article History & Received: December 13, 2020 & Revised: May 26, 2021 & Accepted: June 16, 2021 \\
\hline
\end{tabular}

\section{INTRODUCTION}

Professor Hermann von Helmholtz revolutionised ophthalmology through his invention of the ophthalmoscope in 1851, an instrument which employed the technique of direct ophthalmoscopy [1 - 3]. Soon after, Professor C.G. Ruete modified the device and became the first to implement monocular indirect ophthalmoscopy [1,3,4]. This technique is different from direct ophthalmoscopy as it uses a condensing lens to view the fundus, thus producing an inverted aerial image between the lens and the examiner [5 - 7]. In 1861, the binocular version of this instrument was introduced by Frenchman Felix Giraud-Teulon [3, 5]. Today, the binocular indirect ophthalmoscope (BIO) has become clinically successful as it allows for stereoscopic vision and a wider field of view $[5,7]$.

Over time, companies such as Keeler Ltd (Keeler Ltd, Windsor, UK), Welch Allyn (Hill-Rom Inc), and Heine Optotechnik (HEINE Optotechnik, Munich, Germany) have launched varying models of the BIO headset including wireless headsets and the compact Spectra Iris. Typically, these BIO headsets cost between USD $\$ 1,000$ and USD $\$ 4,000$, rendering them niche equipment and non-equitable to resource-poor regions. In light of this, we aim to discuss an inexpensive technique of performing binocular indirect ophthalmoscopy without a BIO headset. This minimalistic method is useful when the examiner only requires a wide angle view of the posterior fundus.

The BIO works on the principles of refraction and reflec

* Address correspondence to this author at University of Otago Medical School, Dunedin, New Zealand; Tel: +64273355024; E-mail: aqeedasingh@gmail.com -tion, with the use of a strong condensing lens ranging from $13 \mathrm{D}$ to $30 \mathrm{D}[5,7]$. The condensing lens is used to refract the light passing from the illumination source onto the patient's fundi, which then reflects back through the condensing lens to produce an aerial image of the fundus $[6,8]$. To view the image, the examiner's visual axes must be aligned with the aerial image produced [9].

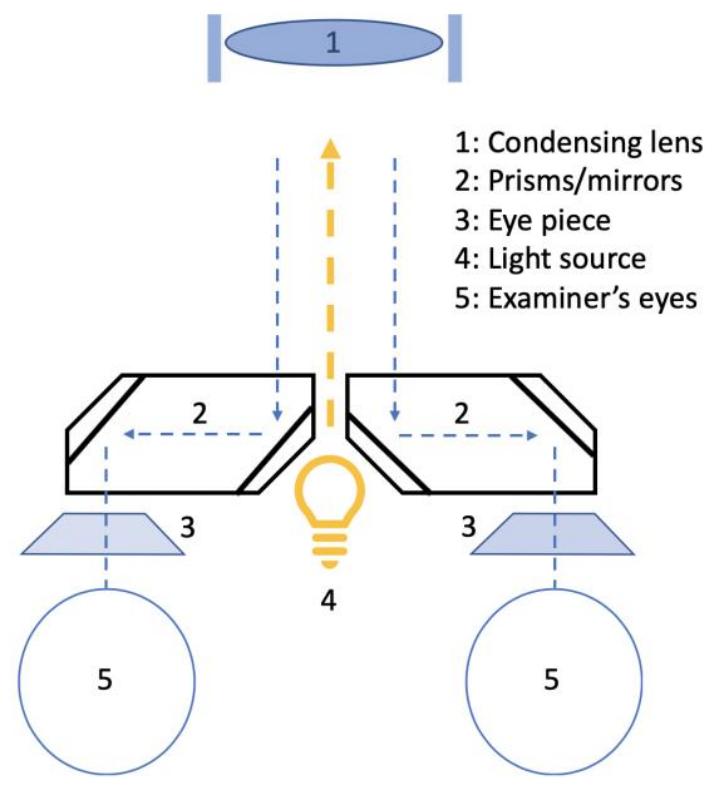

Fig. (1). Binocular indirect ophthalmoscope headset: this figure depicts the optics of the headset. 
In addition to providing co-axial illumination, the purpose of the BIO headset is to reduce the interpupillary distance (IPD) [9]. An IPD of less than $38 \mathrm{~mm}$ makes it easier to fit the view of both the examiner's eyes within the exit pupil of the condensing lens (Fig. 1) [5].

\section{METHODS}

However, to inexpensively conduct this without a BIO headset, the examiner requires a light source such as the direct ophthalmoscope or a pen torch, and a condensing lens such as the US\$10 oDocs 20D disposable lens (oDocs Eye Care Ltd, NZ) or a US\$20 20D Katena single-use lens (Katena Products Inc, USA). It is to be noted that this technique requires dilated pupils. To view the aerial image, the examiner needs to increase the working distance, which can be done by extending the arm and having a degree of ocular accommodation. Consequently, most practitioners can fit the view of both eyes within the exit pupil of the condensing lens to see the image (Fig. 2).

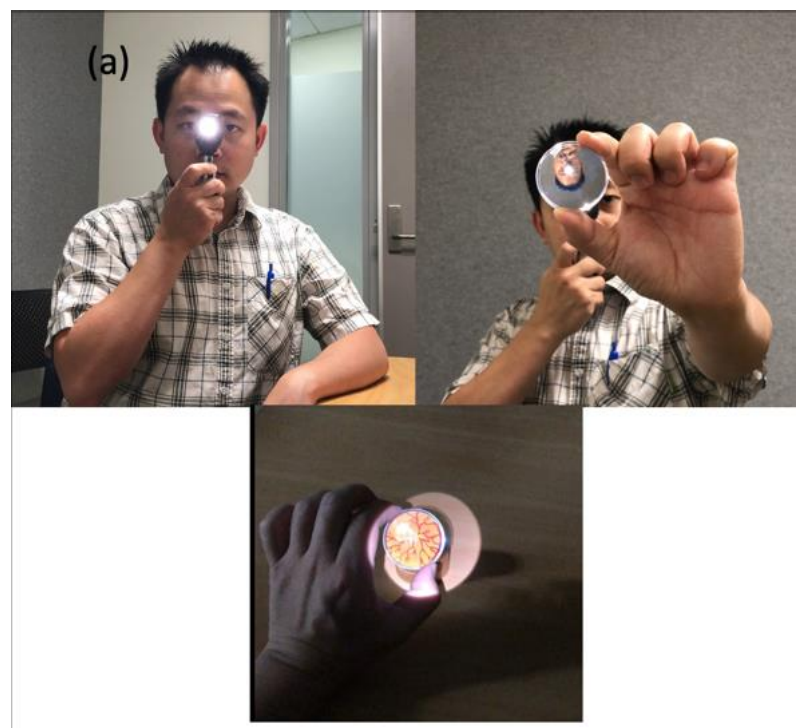

(b)
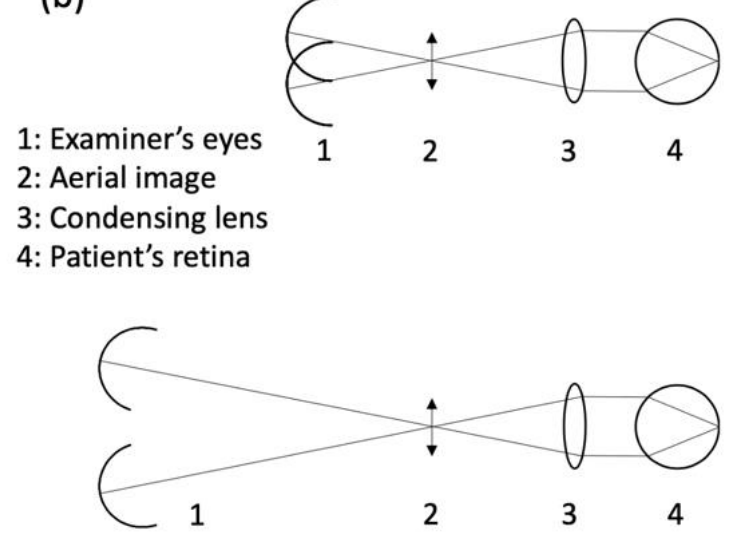

Fig. (2). Mechanism: (a) shows how the illumination should be held, (b) shows how increasing the working distance combined with ocular accommodation allows the examiner to view the aerial image. Consent was obtained from the volunteer to use this photo.

\section{RESULTS AND DISCUSSION}

The primary advantage of this technique is that it allows for stereoscopic visualization of the fundus in the absence of cumbersome and expensive equipment. It has been described previously for monocular indirect ophthalmoscopy, but this does not allow for stereoscopic vision [10].

Having an increased working distance with disposable equipment may also be considered advantageous when examining infectious patients, but this technique may have a steeper learning curve, and some examiners may find it less comfortable fully extending the arm.

\section{CONCLUSION}

Lastly, additional functions cannot be used which are on the commercialised BIO headsets, such as having extra magnifications or filters. However, this inexpensive technique should be able to satisfactorily inform the examiner about the state of the patient's posterior fundus in resource-poor settings or the absence of a BIO headset.

\section{ETHICS APPROVAL AND CONSENT TO PARTICIPATE}

Not applicable.

\section{HUMAN AND ANIMAL RIGHTS}

Not applicable.

\section{CONSENT FOR PUBLICATION}

Consent was obtained from the volunteer to use this photo.

\section{AVAILABILITY OF DATA AND MATERIALS}

Not applicable

\section{FUNDING}

Dr. Hong reports non-financial support from ODOCS EYE CARE LIMITED.

\section{CONFLICT OF INTEREST}

The author declares no conflict of interest, financial or otherwise.

\section{ACKNOWLEDGEMENTS}

Declared none.

\section{REFERENCES}

[1] Sherman SE. The history of the ophthalmoscope. Doc Ophthalmol 1989; 71(2): 221-8.

[http://dx.doi.org/10.1007/BF00163473] [PMID: 2663401]

[2] Tan SY, Shigaki D. Hermann von Helmholtz (1821-1894): inventor of the ophthalmoscope. Singapore Med J 2006; 47(5): 359-60. [PMID: 16645682]

[3] Keeler CR. The ophthalmoscope in the lifetime of Hermann von Helmholtz. Arch Ophthalmol 2002; 120(2): 194-201. [http://dx.doi.org/10.1001/archopht.120.2.194] [PMID: 11831923]

[4] Simonsz HJ. Christian Theodor Georg Ruete: the first strabismologist, coauthor of listing's law, maker of the first ophthalmotrope and inventor of indirect fundoscopy. Strabismus 2004; 12(1): 53-7. [http://dx.doi.org/10.1076/stra.12.1.53.29011] [PMID: 15255369]

[5] Cordero I. Understanding and caring for an indirect ophthalmoscope. 
Community Eye Health 2016; 29(95): 57.

[PMID: 28289323]

[6] Principles of Ophthalmoscopy 2016.https://entokey.com/principles-ofophthalmoscopy/\#R11-V1-63

[7] Binocular Indirect Ophthalmoscopy 2021.https://eyewiki.aao.org/ Binocular_Indirect_Ophthalmoscopy

[8] Garston MJ. The binocular indirect ophthalmoscope (BIO). J Am
Optom Assoc 1977; 48(11): 1403-7.

[PMID: 411815]

[9] Sonia Rani John MC. Arup Chakrabarti. Binocular Indirect Ophthalmoscope. Kerala Journal of Ophthalmology 2009; XXI(4): 419-20.

[10] Bishai KR. An inexpensive method of indirect ophthalmoscopy. Br J Ophthalmol 1989; 73(3): 235-6.

[http://dx.doi.org/10.1136/bjo.73.3.235] [PMID: 2706215]

\section{(C) 2021 Singh et al.}

This is an open access article distributed under the terms of the Creative Commons Attribution 4.0 International Public License (CC-BY 4.0), a copy of which is available at: https://creativecommons.org/licenses/by/4.0/legalcode. This license permits unrestricted use, distribution, and reproduction in any medium, provided the original author and source are credited. 